\title{
Research Report \\ Strategy for the Design of Custom cDNA Microarrays
}

\author{
Matthias G.O. Lorenz ${ }^{1}$, Lizette \\ M. Cortes ${ }^{2}$, Juergen J. Lorenz ${ }^{3}$, \\ and Edison T. Liu ${ }^{4}$ \\ ${ }^{1}$ National Cancer Institute, \\ Gaithersburg, MD, USA; ${ }^{2}$ Uni- \\ versity of Calgary, Calgary, AB, \\ Canada; ${ }^{3}$ National Institute of \\ Neurological Disorders and \\ Stroke, Bethesda, MD, USA; \\ and ${ }^{4}$ Genome Institute of Singa- \\ pore, National University of \\ Singapore, Singapore
}

\section{ABSTRACT}

DNA microarrays are valuable but expensive tools for expression profiling of cells, tissues, and organs. The design of custom microarrays leads to cost reduction without necessarily compromising their biological value. Here we present a strategy for designing custom cDNA microarrays and constructed a microarray for mouse immunology research (ImmunoChip ${ }^{T M}$ ). The strategy used interrogates expressed sequence tag databases available in the public domain but overcomes many of the problems encountered. Immunologically relevant clusters were selected based on the expression of expressed sequence tags in relevant libraries. Selected clusters were organized in modules, and the best representative clones were identified. When tested, this microarray was found to have minimal clone identity errors or phage contamination and identified molecular signatures of lymphoid cell lines. Our proposed design of custom microarrays avoids probe redundancy, allows the organization of the chip to optimize chip production, and reduces microarray production costs. The strategy described is also useful for the design of oligonucleotide microarrays.
\end{abstract}

\section{INTRODUCTION}

DNA microarrays (1) are expensive tools and are largely limited to arrays that have been printed from predefined, commercial sets or that have been constructed from discrete, experimental libraries tailored to specific cell types (2). However, these approaches provide high-cost microarrays containing only basic genes or a snapshot of the expressed sequence tags (ESTs) present at the time and conditions of library sampling. A custom design of microarrays reduces costs and allows the selection of relevant genes of complex organ systems such as the immune system.

Rich resources for the construction of microarrays are EST databases. Currently, more than $1.2 \times 10^{7}$ ESTs (August 23,2002 ) from cDNA libraries derived from diverse cells and tissues for various model organisms are available for the construction of specialized arrays. Algorithms, such as Unigene, automatically assemble these ESTs into clusters that tentatively define distinct genes. Additional information for each cluster, such as the tissue types in which the gene has been expressed and map location, is attached. However, the selection of genes and their representative clones from the large database remain significant challenges in the construction of custom microarrays. The difficulty is compounded by the high frequency of redundant gene sequences and the need to avoid the selection of clones with incorrect identities or phage contamination (3). Despite these rich resources, a method is missing that would allow the selection of relevant clusters and corresponding clones for the construction of microarrays.

To exploit the potential of EST databases for the generation of custom microarrays, we have developed a strate- gy to construct nonredundant and organized arrays. Here we used this strategy to design a microarray for mouse immunology research (ImmunoChip ${ }^{\mathrm{TM}}$ ). The Unigene database was used to identify immunological organ-, tissue-, or cell-specific clusters. Since multiple ESTs are present in most Unigene clusters, a strategy was developed to identify the best representative clone for each of the selected immunologically relevant clusters. The proposed strategy is also applicable for the design of custom oligonucleotide microarrays.

\section{MATERIALS AND METHODS}

\section{Microarray Construction}

Cluster selection. The mouse Unigene database built 75 was used for the construction of the microarray. A clone was selected if it was derived from one of the following immunological murine libraries: primary lymphoid organ [fetal liver (LID139, LID233, LID287, LID221), thymus (LID26, LID28, LID58, LID296, LID299, LID302), and bone marrow (LID113, LID129, LID130, LID140, LID142)]; secondary lymphoid organ [spleen (LID63, LID152, LID159, LID172, LID201) and lymph node (LID66)], or an immunocyte [B-cell (LID366), T-cell (LID50), macrophage (LID5, LID12, LID20, LID47, LID82), stem cell (LID32, LID99), and lymphocyte (LID9)]. The library LID366 was derived from Unigene built 76 . Cluster IDs were electronically added, and a cluster selection list was generated.

Clone selection. Clusters were organized in gene module (GM), homologous gene module (HM), and EST module (EM). Reference genes [LocusLink 
(RefSeq, 04/03/00)] for GM and cluster representatives (Unique.seq, Unigene) for HM were added, verified, and blasted against NCBI nonredundant database (Mus musculus restricted) [BLAST 2.0 Network client (blastcl3.hqx) (E: 4e49)]. Result files were reformatted, ImmunoChip score (sum BLAST score, $5^{\prime}$ position of alignment) for GM was calculated, and a ranked list was generated. For EM, a priority list was generated in the following order: LID50 > LID366 > LID47 > LID99 > LID63 > LID154 > LID66 > LID58 > LID296 > LID299 > LID26 > LID221 > LID287 > LID142. Clones were removed from the priority list if reported in the IMAGE consortium problematic database.

Glass slide coating. Microscope slides (Gold Seal, Portsmouth, NH, USA) were cleaned in $3.6 \mathrm{M} \mathrm{NaOH}$, $70 \%$ ethanol for $2 \mathrm{~h}$ and washed with double-distilled water. Glass slides were coated with $0.01 \%$ poly-L-lysine (Sigma, St. Louis, MO, USA) $(10 \%$ PBS solution) for $1 \mathrm{~h}$, centrifuged, and dried for several weeks.

Clone processing. Bacterial clones (IMAGE clones; Incyte Genomics, Palo Alto, CA, USA) were cultured in 1.5 $\mathrm{mL}$ Superbroth supplemented with 50 $\mu \mathrm{g} / \mu \mathrm{L}$ ampicillin (Invitrogen, Carlsbad, CA, USA) for $12 \mathrm{~h}$ at $37^{\circ} \mathrm{C}$ and 200 rpm without additional colony repurification. Plasmid DNAs were prepared (Wizard $^{\mathrm{TM}}$ SV96; Promega, Madison, WI, USA) by robotics (Biomek ${ }^{\circledR} 2000$; Beckman Coulter, Fullerton, CA, USA). PCR primers (5'-CCCAGTCACGACGTTGTAAAACGACGGCCA-3' and 5'-GATAACAATTTCACACAGGAAACAGCTATG-3') were used for amplification of cDNA inserts in pT7T3-Pac, Bluescript ${ }^{\circledR}$ SK-, Bluescript SK+, Bluescript Sal/Mlu, pCMVSPORT, pCMV-SPORT2, pCMVSPORT6, and pSPORT1 vectors. PCR primers (5'-CGGAAGTGTTACTTCTGCTCTAAAAGCTGC-3' and 5'-CTCTAGACTAGTCTAGCGGCCGCGA CCTG-3') were used for amplification of cDNA inserts in pME18S-FL3 plasmids. The GM, HM, and EM (5 U Hotstart Master Mix; Qiagen, Valencia, CA, USA) were amplified (Peltier Thermal Cycler; MJ Research, Waltham, MA, USA) according to the manufacturer's instructions. The PCR conditions were $95^{\circ} \mathrm{C}$ for $5 \mathrm{~min}$, fol- lowed by 32 cycles of $95^{\circ} \mathrm{C}$ for $30 \mathrm{~s}$, $59^{\circ} \mathrm{C}$ for $30 \mathrm{~s}$, and $72^{\circ} \mathrm{C} 2.5 \mathrm{~min}$, with a final extension of $72^{\circ} \mathrm{C}$ for $10 \mathrm{~min}$. PCR products were cleaned up by filtration (Multiscreen ${ }^{\mathrm{TM}}$ PCR; Millipore, Bedford, MA, USA) and resolved in water. All PCR products were checked by agarose-gel electrophoresis $(<5 \%$ with double bands) and replated in 384well plates (Nalge Nunc International, Rochester, NY, USA). For printing, the PCR products were spotted as a $3 \times$ SSC solution on lysine-treated glass slides with $175 \mu \mathrm{m}$ spacing by using a 32-pin (TeleChem International, Sunnyvale, CA, USA) spotter robot (OmniGrid ${ }^{\circledR}$; GeneMachines, San Carlos, CA, USA). For post-processing, the printed glass slides were dried overnight and blocked with 1-methyl-2-pyrrolidone/boric acid (pH 8.0) (Sigma).

Phage screen and sequence analysis. Bacterial clones were tested for the presence of phages using the JH101 bacteria strain (Stratagene, La Jolla, CA, USA). Sequencing was carried out on plasmids (Turbo; Qiagen) using BigDye $^{\mathrm{TM}}$ terminator chemistry (Applied Biosystems, Foster City, CA, USA) in both directions (T7, T3) (Lark Technologies, Houston, TX, USA). Derived sequences were judged [Chromas 2 (Technelysium)], trimmed, and blasted against the murine dbEST database under stringent conditions [BLAST 2.0 Network client (blastcl3.hqx (E: 4e-49)]. Of the clone sequences, $95.1 \%$ corresponded to the selected clone identity.

Cell lines and cell separation. Cell lines were obtained from ATCC (Manassas, VA, USA) and cultured according to the provided instructions.

RNA preparation and labeling. Total RNA was prepared using TRIzol ${ }^{\circledR}$ reagent (Invitrogen), labeled with $\mathrm{Cy}^{\mathrm{TM}} 3$-dUTP, Cy5-dUTP by reverse transcription, and hybridized as described previously (4).

\section{Data Analyses}

The microarray was scanned at 10 $\mu \mathrm{m}$ pixel size, gridded, and analyzed (GenePix ${ }^{\circledR} 4000 A$; Axon Instruments, Union City, CA, USA). Background was subtracted, and the median sum and median ratio were calculated. Flagged spots and spots with sum intensity (CH1, CH2) less than $100 \mathrm{AU}$ 


\section{Microarray Technologies}

were excluded. Data were normalized by trimmed mean at $10 \%$ to account for differences in the amounts of RNA labeled or labeling efficiencies.

\section{RESULTS}

In the present study, we describe a strategy developed to design a custom cDNA microarray. Here we designed, physically constructed, and validated a microarray for mouse immunology research.

\section{STRATEGY FOR THE DESIGN OF MICROARRAYS}

\section{Identification of Immunologically \\ Relevant Clusters}

The immune system consists of many different cell types, tissues, and organs. We designed an immunologically relevant microarray containing probes of genes expressed in immunocytes, immunological tissues, and lymphoid organs in two steps. The first step was to select immunological clusters based on the expression of their cluster members in immunological libraries. We defined libraries from primary lymphoid organs (fetal liver, thymus, and bone marrow), secondary lymphoid organs (spleen and lymph node), and immunocytes (stem cell, B-cell, T-cell, and macrophage) as immunologically relevant. In total, 31 of 207 Unigene libraries were selected. In addition, immunologically relevant genes identified through a knowledge-based search of the existing literature were added. The literature searches were either of existing databases (LocusLink) or journal publications (modular gene lists) and performed by trained immunologists. A total of 67,359 records from Unigene databases, 3415 records from LocusLink searches, and 618 genes from the modular gene list searches were selected (Figure 1A). Since the resulting list was highly redundant, it was reduced to a nonredundant gene set by organizing cDNAs and genes into mouse Unigene clusters (Figure 1A). We identified 16,749 unique clusters. This set of clusters approximates the full complement of genes expressed by the immune system.

\section{Selection of Representative Clones from Each Immunological Cluster}

Most clusters contain multiple representative clones; therefore, the next step was the selection of the best representative clone for each cluster that would be present on the microarray (Figure 1B). The clusters fell into three categories of sequences: $(i)$ those that were identified as known murine genes, with associated gene name and sequence, were grouped into the GM; (ii) those whose sequences were highly ho- 
mologous $(>90 \%)$ to known genes in other organisms but for which the mouse gene has not been characterized were assigned to the HM; and (iii) those sequences with no or only moderate or weak homology to known genes - thus those that did not fit into either of the other two modules-were placed in the EM.

Different selection criteria for the identification of the best representative clone within a cluster were applied to each of the three modules, as follows.

GM. A reference gene for each cluster was obtained from LocusLink. $\mathrm{Cu}$ rated reference genes were preferred over un-curated ones. To prove the integrity of the selected cluster, the reference gene for each cluster was verified by performing a BLAST search against the murine NCBI nonredundant database. In those few cases where the selected reference gene did not represent the cluster appropriately, the cluster was analyzed and an appropriate reference gene was selected manually.

To determine the clones within a cluster, reference gene sequences were blasted against the murine EST clone sequences under stringent conditions (E: 4e-49). To select the best representative clone from among the homologous EST clones, an ImmunoChip score was calculated as the sum of the BLAST score and the $5^{\prime}$ position of alignment between the reference gene and each individual EST. This unweighted ImmunoChip score was sufficient to give a high score to ESTs, which have a good alignment to their reference gene on the most 3' part of the gene. ESTs in each cluster were ranked according to the score (priority list).

HM. Genes in the HM do not have a M. musculus reference gene. As a substitute, the longest EST clone within a cluster was chosen as the reference sequence, which was then compared against the murine EST database by a BLAST search. Again, ESTs in each cluster were ranked based on their BLAST score. Although the reference EST will always have the highest BLAST score within the cluster, the remaining clones could also be ranked.

EM. Most clusters in the EM were singletons or had clusters with only a few records. ESTs in these clusters tend to give only moderate scores if blasted against each other. The generation of a meaningfully ranked list based on these scores was questionable. To overcome this problem, we decided to pick ESTs that were physically derived from immunological libraries. A ranked list was then generated according to the origin of the library: lymphoid cell type over secondary lymphoid organs over primary lymphoid organs. Only ESTs with a reported sequence longer than $100 \mathrm{bp}$ were considered.

\section{Clone List Construction}

The ranked clones of each module were then checked against the IMAGE consortium database of problematic clones. Clones reported to be problematic were removed from the ranked list. Among the remaining nonproblematic clones, those with the highest rank of each cluster were selected as representatives of the cluster and used in the con- struction of the microarray. In those cases where a cluster was represented only by problematic clones, the cluster was not used at all in the chip construction.

\section{Duplicate Merging and Subclassification}

Using the strategy outlined above, a total of 13,385 unique IMAGE clones, each presumably representing a different gene, were re-arrayed from the IMAGE clone collection. A few duplicate clones were detected at this point, which were merged to the next higher module. The reason for duplicate clones could be that the Unigene algorithm organizes EST sequences in clusters without using clone information. Thus, EST sequences $\left(5^{\prime}\right.$ or $3^{\prime}$ sequences) derived from the same clone can get distributed into different clusters. Another possibility is the generation of chimeric EST clones during li- 


\section{Microarray Technologies}

brary construction. Clones were also checked for availability. When the clone with the highest rank was not available, the next clone on the ranked list was substituted. To compensate for known differences in growth rate of bacterial hosts, selected clones were physically separated into slow-growing and fast-growing bacterial plates. Thus, each module of the microarray was re- arrayed in sections according to bacterial hosts. The DH section contains IMAGE clones of the bacterial strains DH10B, DH12S, and GeneHog DH10B. The SOLR section contains

A

\begin{tabular}{|c|c|c|c|}
\hline \multicolumn{2}{|l|}{ Expression } & \multicolumn{2}{|l|}{ Literature } \\
\hline Unigene $757175(20$ & 02 libraries) & Databases & Modular Gene Lists \\
\hline $\begin{array}{l}\text { Organ/Tissue/Cell } \\
\text { Bone Marrow } \\
\text { Thymus } \\
\text { Fetal Liver (d13-d14) } \\
\text { Spleen } \\
\text { Lymph Node } \\
\text { Stem Cell } \\
\text { T-cell } \\
\text { B-cell } \\
\text { macrophage }\end{array}$ & $\begin{array}{l}\text { Library ID } \\
142,130,129,113,140 \\
52,299,296,26,28,302 \\
233,287,221,139 \\
63,201,154,159,172 \\
66,9 \\
99,32 \\
50 \\
366 \\
47,12,20,82,5\end{array}$ & $\begin{array}{l}\text { LocusLink (11980 records) } \\
\text { Entrez } \\
\text { PubMed } \\
\text { OMIM }\end{array}$ & $\begin{array}{l}\text { Basic } \\
\text { CD/Ly Marker } \\
\text { Cytokines/Chemokines } \\
\text { Signal Transduction } \\
\text { Apoptosis } \\
\text { MHC pathway } \\
\text { Oncogene/Tumor } \\
\text { Suppressor }\end{array}$ \\
\hline 67359 records selecte & $\begin{array}{r}\text { Redundant } \\
-713 \\
\begin{array}{r}\text { Non-Red } \\
-167\end{array}\end{array}$ & $\begin{array}{l}\text { ne/EST List } \\
\text { lected - } \\
\text { chment of Cluster IDs } \\
\text { luster List } \\
\text { lected - }\end{array}$ & 618 records selected \\
\hline
\end{tabular}

B

Selected "Immuno" clusters -16749 clusters -

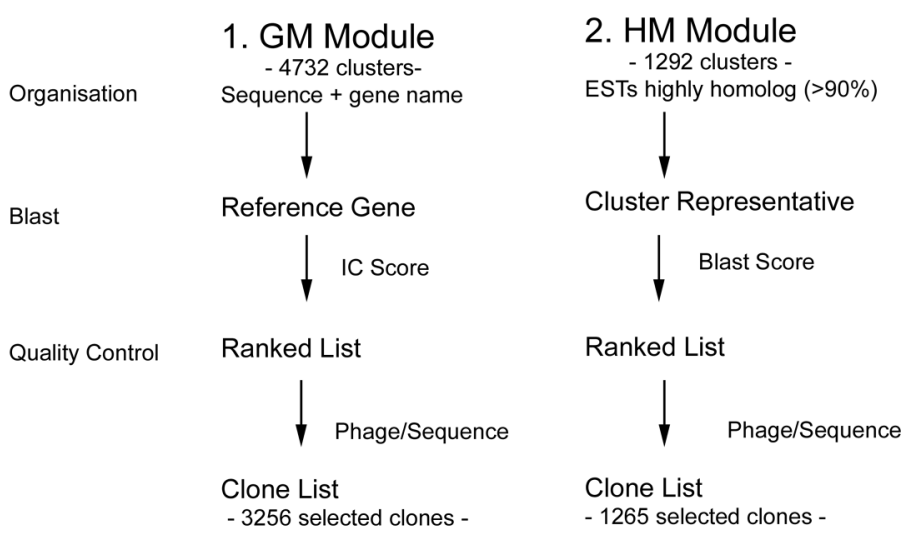

3. EM Module - 10725 clusters moderate, weakly or not homolog

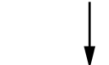

All Cluster Members Cell>sec. Organ > prim. Organ

Ranked List

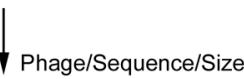

- 3256 selected clones -

- 1265 selected clones -

Clone List

8864 selected clones -

Figure 1. Construction of the ImmunoChip. (A) Identification of immunologically relevant clusters. The microarray was constructed by selecting sequences expressed in immunological organs and cells (Unigene built 75) and complemented by genes with reported immunological function (LocusLink, Pubmed, Entrez, and OMIM). Cluster information was attached to all sequences and duplicates removed. (B) Module classification and selection of the best representative clone for each cluster. Immunological clusters were categorized into the gene module (GM), homologous gene module (HM), and EST module (EM). Based on the presence of a cluster in a specific module, different strategies were chosen to select the best representative clone for each cluster. GM, a reference gene was identified and blasted against the dbEST database. An ImmunoChip (IC) score was calculated based on BLAST score and position of 3' alignment. HM, the longest EST was defined as cluster representative and blasted against dbEST. EM, all members of a cluster were ranked based on their presence in immunological libraries in the following order: cell $>$ secondary lymphoid organ > primary lymphoid organ. For all modules, priority lists were generated, and reported problematic clones were removed. The clone with the highest rank was chosen as the best representative for the selected cluster. 


\section{Microarray Technologies}

the slow-growing SOLR bacterial strain. In addition, the plates were logically divided into M13 and custom partitions according to plasmid type. Both strategies allowed the optimized use of bacterial plates and plasmid plates during microarray production.

\section{Summary of Microarray Construction}

The strategy described above yielded 16,749 identified immunological relevant clusters, 13,385 selected best representative clones, and 13,208 reracked clones. During the clone selection, the GM size was reduced from 4732 to 3256 mostly because of missing sequences for already named (but uncloned) genes. The HM remained stable (from 1292 to 1265). The EM was reduced from 10,725 to 8864 mostly because of the presence of large numbers of singletons, which eliminated the option of using substitutes. During the re-racking, 177 clones were not available. The re-racked HM gained 31 clones because of the merging of duplicates and the reorganization of clusters to modules at the higher level. The physically re-racked chip contains 3202 known genes, 1296 homologous genes, and 8730 unknown ESTs for a total of 13,208 clones. While 12,956 clones $(98.1 \%)$ were selected based on expression patterns in immunological libraries, only 252 clones $(1.9 \%)$ were selected based on literature searches.

\section{Validation of the Microarray}

Two validation approaches were used to assess the microarray: individual spot validation and whole-chip validation. Single spots were validated by testing for phage contamination and by direct DNA sequencing. The individual spot validation test revealed that no phage could be detected out of five GM, five HM, and five EM 96-well plates and that $95.1 \%$ of the readable clones were correct.

The integrity of the entire microarray was assessed by hybridization with RNA derived from a variety of B cell, $\mathrm{T}$ cell, and macrophages cell lines. RNA from the fibroblast cell line SVT2 was used as a reference. Molecules whose level of expression was in- creased or decreased more than 3-fold, relative to the fibroblast reference, were considered to be significantly different from expression in fibroblasts. Using this definition, the microarray identified the expected molecular signatures of each of the cell types. Known markers for mature B-lymphocyte include CD19, CD20, CD24, CD37, Fc receptors, complement receptors, major histocompatibility complex (MHC) class I, and MHC class II molecules. The microarray identified in the EL-4 T cell line the T cell receptor TCRa, CD28, CD3 $\delta, C D 3 \gamma$, and the Tcell-specific signal transduction molecules ZAP70 and Lck as EL-4 markers. In addition, the MHC class I molecules H2-K, H2-Q2, H2-L, H2-D, and $\beta 2$-microglobulin were more than 2-fold up-regulated in EL-4 T cells. The cell line RAW264.7 represents a monocyte/macrophage state, which expresses a high level of lysozymes. As measured by the microarray, RAW264.7 highly overexpress the lysozymes $\mathrm{P}$ (Lzp-s) and $\mathrm{C}$ type $\mathrm{M}$ (Lzm), the macrophage colony-stimulating factor 1 receptor (Csfr1), and macrophage expressed gene 1 (Mpeg1).

\section{DISCUSSION}

Here we described a strategy for the design of custom cDNA microarrays. We developed and used a strategy that interrogates EST databases available in the public domain. Clusters were selected in silico based on the expression of their members in relevant libraries and complemented by further selection from literature searches. Selected clusters were organized in modules, and the best representative clones were identified. Our proposed design of custom microarrays avoids probe redundancy, reduces microarray production costs, and allows the organization of the chip because of its EST properties (genes, homologous genes, and nonhomologous genes), vector, and bacterial hosts (fastand slow-growing bacteria strains).

Although the outlined strategy was developed for the design of cDNA microarrays, it can also be used for the design of oligonucleotide arrays. For the design of custom oligonucleotide ar- rays, relevant clusters could be identified as outlined above. The best representative gene sequence for each selected clusters would be ideally a reference gene (Reference Gene Database, $\mathrm{NCBI}$ ). If a reference gene is not available, then the best representative clone could be identified by the clone selection strategy. The identified best representative sequences could then be used for identification of the best representative oligonucleotide probe on the chip.

\section{ACKNOWLEDGMENTS}

This work was supported by the intramural program of the National Cancer Institute. The authors thank Drs. Jeff Green, Richard Hodes, and Dinah Singer for critical reading of the manuscript.

\section{REFERENCES}

1.Freeman, W.M., D.J. Robertson, and K.E. Vrana. 2000. Fundamentals of DNA hybridization arrays for gene expression analysis. BioTechniques 29:1042-1055.

2.Tanaka, T.S., S.A. Jaradat, M.K. Lim, G.J. Kargul, X. Wang, M.J. Grahovac, S. Pantano, Y. Sano, et al. 2000. Genome-wide expression profiling of mid-gestation placenta and embryo using a 15,000 mouse developmental cDNA microarray. Proc. Natl. Acad. Sci. USA 97:9127-9132.

3.Halgren, R.G., M.R. Fielden, C.J. Fong, and T.R. Zacharewski. 2001. Assessment of clone identity and sequence fidelity for 1189 IMAGE cDNA clones. Nucleic Acids Res. 29:582-588.

4.DeRisi, J.L., V.R. Iyer, and P.O. Brown. 1997. Exploring the metabolic and genetic control of gene expression on a genomic scale. Science 278:680-686.

Received 24 October 2002; accepted 25 March 2003.

Address correspondence to:

Dr. Matthias Lorenz

Navy Medical Research Center

503 Robert Grant Avenue

Silver Spring, MD 20910, USA

e-mail:lorenzm@nmrc.navy.mil 